\title{
Skin Lesions Detection using Meta-Heuristic Method
}

\author{
Mohanad Hasan Ali Aljanabi* \\ Department of Electrical Power Techniques Engineering, Al-Furat Al-Awsat Technical University, Iraq
}

Received: 制: September 15, 2018; Published: 制 September 26, 2018

*Corresponding author: Mohanad Hasan Ali Aljanabi, Department of Electrical Power Techniques Engineering, Al-Furat Al-Awsat Technical University, Iraq

\begin{abstract}
Melanoma skin cancer has been one of the quickest uprisings of totally cancers, which has a high hazard of prevalence. This deadliest form of melanoma must be detected premature for effective handling. In this work, a technique was used to aid the premature detection of skin cancer lesions. For the segmentation to be considered correct using the Artificial Bee Colony (ABC) method, the result obtained was compared with the segmentation of the dermatologist. This method is applied on dermoscopy images were obtained of the PH2 database. The algorithm is one of the foremost widespread techniques to obtain infinite chances to solve the $\mathrm{ABC}$ rule that provides accurate results in the quickest possible time. The artificial bee colony algorithm recognizes whether moles are melanoma or not and at any stage of danger also the results are compared with the results from the existing algorithm of melanoma detection; it achieved good results in the conditions of high specificity, accuracy and sensitivity $(92.50,97.20,93.02) \%$. The $\mathrm{ABC}$ algorithmic is effective and improves early detection with high accuracy for skin lesions leads to decrease in death rates.
\end{abstract}

Keywords: ABC; Image Segmentation; Melanoma; Dermoscopy; Lesion; Meta-heuristic

\section{Introduction}

Meta- heuristic methods optimization algorithm is depending on contact and cooperation between individuals in a set of organisms [1]. In USA in 2013, there was more than 76,000 persons are diagnosed with skin cancer and $12 \%$ would die. The current position is that malignant melanoma occurrence rates are very high. From 1992 until the present day, research concerning the Malignant Melanoma (MM) found that occurrence average for nonHispanic white men and women were rising yearly rate of roughly ten percent. If malignant is noticed near the beginning, where it is categorized at stage $\mathrm{I}(\mathrm{t}=0.76 \mathrm{~mm})$, the past 5 years rate of survival is ninety-six percentages. In the opposite, rate of survival reduced to $4 \%$ for the past five years, if the malignant was in Phase IV [2,3]. The ACSs evaluates for malignant melanoma in the USA for 2018 are about 91,270 new malignant cases will be detected $(55,150$ in males and 36,120 in females), and the number 9,320 persons are predictable to die of MM (5,990 males and 3,330 females) [4]. Barata et al. [5] used color constancy to improve dermoscopy image categorization for skin lesions detection method.

In [6] the intuitive mining feature from two different databases images has been studied using machine learning method. In [7], established a multi-model of semantic segmentation by FCN that can segment three samples of skin lesions. Yuan et al. [8] introduced automatically segmentation of skin lesions utilizing deep Convolutional-Deconvolutional Neural Networks (CDNN) then the work is developed to two-stage; the technique of segmentation and categorization of melanoma lesions. Al-masni et al. [9] used various dermoscopy databases for skin lesions Full Resolution Convolutional Network (FrCN) to show better performance compared with existing methods of melanoma detection that leads to an enhancement in the segmentation analysis [9]. The results established that the Social Group Optimization (SGO) is very effective to extract lesion sectors from hairy skin tumor images compared with the alternatives studied of detection skin lesions [10]. Li \& Shen [11] are applied new technique to detect three types of skin cancer lesions by using deep learning system to distinguish between melanoma and benign.

Guo et al evaluated the results validate of the Neutrosophic clustering and adaptive region growing methods on 500 images to achieve better performance for detection the skin lesions when compared with another segmentation method [12]. Abbes \& D Sellami [13] searched for bag of words (FoW) model in semantic annotation utilizing machine learning (SVM) to develop the automatic skin lesion categorization. For using knowledge transfer approach of skin tumors screening by deep learning machine to reach high specificity, the results suggest that the experimental design is sensitive to the choice of lesions positive or negative classes [14]. Xie et al. [15] developed a new algorithm with a Self-Generating Neural Network (SGNN) and features descriptive of tumor color to get high accuracy for classified model is melanomas case or benign case [15]. The best combination of features, depended on shape 
properties, colour variation and texture analysis, by using different feature extraction methods it can be extracted, by an optimum path forest classifier promising results are achieved Mahdiraji et al. [16] worked in the same field of color variation of the skin lesion images, acquired with standard cameras computational methods are introduced based on Multilayer perceptron neural network, the results are best compared with results of DermIs and Derm Quest databases [16].

The $\mathrm{ABC}$ algorithm receives input from the segmented image of the skin lesion, the proposed model improves existing methods in the field of detection skin tumors to support the doctors to get the correction decision after segmentation and final detected image compared with ground truth. On the way to skin cancer lesions detection was $\mathrm{ABC}$ algorithm to help contributors develop image examination devices to enable the automated analysis of malignant melanoma from dermoscopic images. We modify and extend the $A B C$ segmentation method. Based on the factor of the skin lesion, the $\mathrm{ABC}$ algorithm found the optimum threshold value for the detection of the melanoma from the skin lesion. Despite the good results previously presented, the proposed technique suggests a problem of over-adjustment, that is, the statistical model adjusted too closely to the set of samples. And, thus obtaining a better understanding in the detection and aiming the development of software. To be tested in clinics and hospitals, helping to reduce the rate of mortality for this and other cancers.

\section{Methodology of Detection Artificial Bee Colony Algorithm}

Artificial Bee Colony (ABC) algorithm is one of the better, modern nature motivated optimization algorithms improved by intelligent searching performance of honey bee swarms. In the $\mathrm{ABC}$ algorithm, searching honey bees are classified into three sets; specifically, worker bees, onlooker bees and scout bees. The procedure of foraging begins with the scout bees being sent to find food resources in the colony surroundings in a chance distribution [17]. This dance is crucial for the bee communication and describes the food resource direction and nectar (fitness value). The onlooker bees select the most profitable source based on all the information revealed by the waggle dances. The $\mathrm{ABC}$ is a computational intelligence of the procedure of estimation via natural election. The artificial bee colony algorithm is shown in Figure 1 . Then the flowchart of utilized steps by ABC algorithm instituted skin cancer detection as shown in Figure 2. There are three steps for the ABC method.

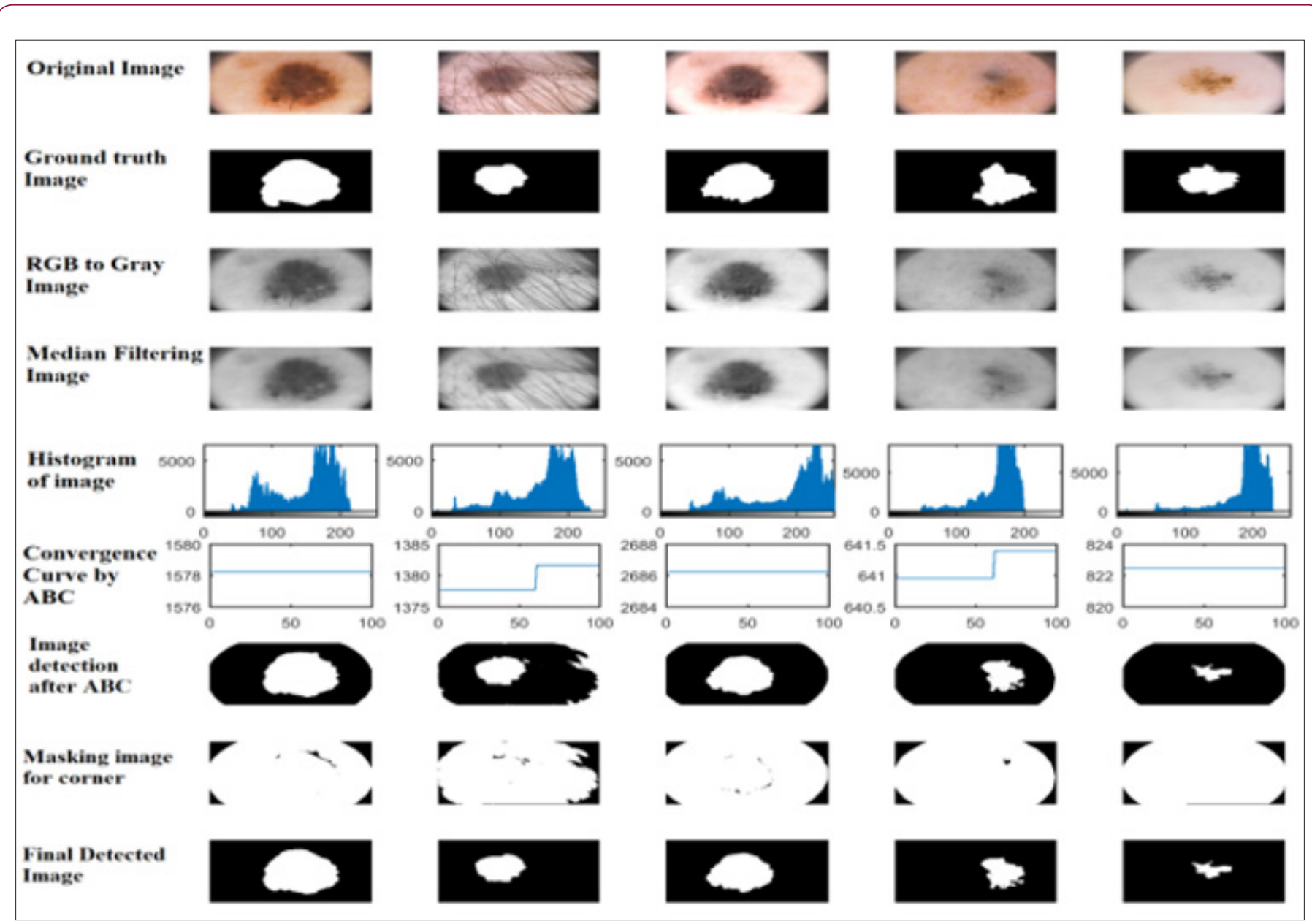

Figure 1: Methodology utilized in artificial bee colony algorithm instituted skin cancer detection. 


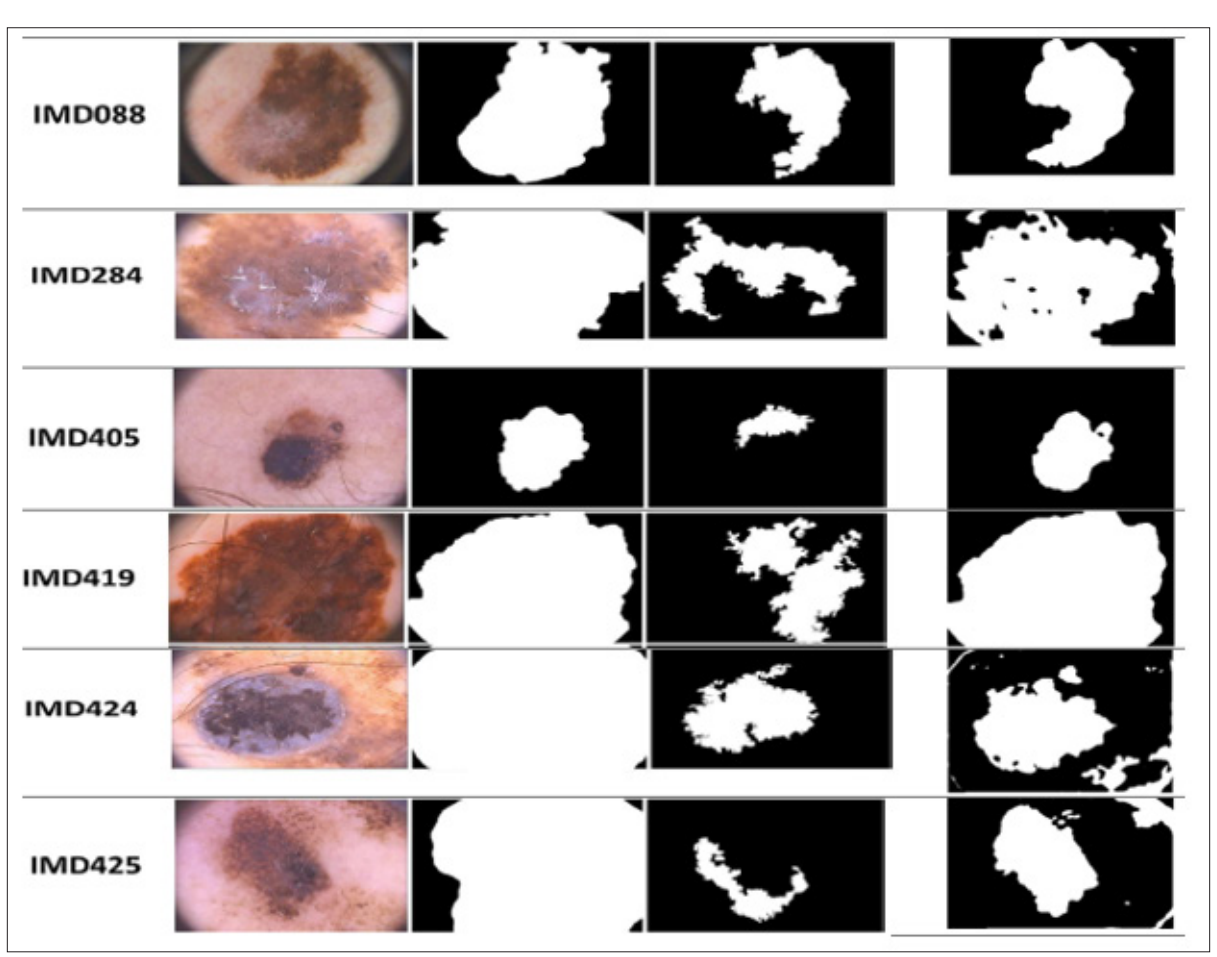

Figure 2: Melanoma detection for proposed method compared with ASLM method.

A-Movement of the Onlookers: Calculation of the new position from equation:

$$
y_{i, j}=L B_{i, j}+r\left(U B_{i, j}-L B_{i, j}\right)
$$

YK: an arbitrarily elected contestant solution, $\mathrm{LB}_{\mathrm{i}, \mathrm{j}}$ The position of the onlooker bee., LB the minimum and UB the maximum limits are the $\mathrm{j}^{\text {th }}$ length size, $\mathrm{r}$ : The randomly chosen worker bee $[-1,1], \mathrm{j}$ : The dimension of the solution.

B-Movement of the Scouts: The movement of the scout bees follows equation (2).

$$
Z_{i, j}=y_{i, j}+\sigma_{i, j} \cdot\left(y_{i, j}-y_{k, j}\right)
$$

$\mathrm{j}$ : an arbitrary length index selected from the group. $\sigma_{i, j}(0,1)$ depend on an ordinary distribution and then the best solution is selected between the food source and their candidate solution.

C-Movement of the Onlookers: Probability of selecting a nectar source:

$$
P_{i}=\frac{F F_{i}}{\sum_{j=1}^{S R} F_{j}}
$$

$P_{i}$ : The probability of choosing the $i_{\text {th }}$ worker be, SR: The number of worker bees, F: The fitness value, yk: Arbitrarily selected candidate solution $(\mathrm{i} \neq \mathrm{k})$. There are numerous advantages for using the $\mathrm{ABC}$ procedure, good updated information to lead the honey bees and the detection of new fields by dance designing. The steps in the artificial bee colony algorithm can be explained as: -

\section{Start}

Initialize the food resource. Find the fitness values for random solution. Onlooker bees select a source depending on the best quality of the solution (1). Find possibility value for every food resource based on equation (2). Assign worker bees as scouts to discover a new food resource. Fresh solution for Onlooker. Update position of the bees it with equation (3). If Requirements criterion met exceeds maximum number of iterations, then after that stop the process and display the output results, Else Repeat to the Step 2. End

In this section, the methodology of melanoma detection depends on the $\mathrm{ABC}$ method as shown in Figure 1. The input data were captured from the 200 RGB PH2 datasets of the Cancer Centre and Institute of Oncology, as dermoscopic images [18]. The steps of all rows of methodology are shown in Figure 1: a-row 1 original image taken from camera or dermoscopy, b- row 2 ground truth images to compared with original image, c- row 3 convert RGB to gray image, $d$ - row 4 using median filter to eliminate noise, erow 5 getting histogram of image, f- row 6 convergence curves by $\mathrm{ABC}$ method. g- row 7 process image detection after $\mathrm{ABC}$, h- row 8 masking images for corner and i- row 9 final steps to detect image. Table 1 shows the performance factors are used from the equations below.

Table 1: The performance factors.

\begin{tabular}{|c|c|}
\hline Measure & Formula \\
\hline Sensitivity & $\mathrm{TP} /(\mathrm{TP}+\mathrm{FN})$ \\
\hline Specificity & $\mathrm{TN} /(\mathrm{FP}+\mathrm{TN})$ \\
\hline Accuracy & $(\mathrm{TP}+\mathrm{TN}) /(\mathrm{TP}+\mathrm{FP}+\mathrm{TN}+\mathrm{FN})$ \\
\hline
\end{tabular}

\section{Results and Discussion}

In view of all the situations, the proposed method for the detection of melanoma presented in this study aims to facilitate 
the detection of this type of cancer in its initial stage and thus, provide the appropriate treatment to the patients. The results are achieved using a MATLAB 2015b version. The total 200 images are processed, and the relevant data is achieved. To compare the effectiveness, the positive predicted value, negative predicted value, accuracy, computation time, specificity and sensitivity are accepted for Analytics. Figure 2. Show the melanoma image processed that prove the validation of the proposed algorithm. In order to verify the results of the proposed method, it is compared with a newly developed method [5-7,9,10,13,19]. Six melanoma images are selected randomly and detection is carried out with both the proposed method and ASLM method from PH2 databases. Figure 2 shows the performance parameter comparison for the 200 images detection utilizing the artificial bee colony compared with another method are using in Table 2. For the effectiveness of the proposed method, it is compared with already presented melanoma detection techniques from Figure 2. with groups of 200 images from databases. From these results, the proposed method has superior parameters in specificity, accuracy and sensitivity $(92.50,97.20,93.02) \%$ but it is not favoured for other considered algorithms. The proposed method has a superior sensitivity property than other considered methods, excluding another method.

Table 2: The performance factors of the proposed method (\%).

\begin{tabular}{|c|c|c|c|}
\hline Methods & SEN & SPE & ACC \\
\hline Proposed method & 92.5 & 97.2 & 93.02 \\
\hline Barata et al. [5] & 90.4 & 97 & 92.8 \\
\hline Al-masni et al. [9] & 93.72 & 95.65 & 95.08 \\
\hline Dey et al. [10] & 92.5 & 76.3 & 84.3 \\
\hline
\end{tabular}

The proposed $\mathrm{ABC}$ method results on $\mathrm{PH} 2$ database images of malignant lesions, shows that IMD088 (blue-whitish veil, streaks and regression areas), IMD284, IMD405 and IMD419 (blue-whitish veil, for second, third and fourth row), IMD424 (blue-whitish veil and streaks) for fifth row, and IMD425 (bluewhitish veil and regression areas) for sixth row. All parameters were calculated utilizing a stratified of cross validation method, in which the databases are divided into three subgroups, each with approximately the numbers of 40 unhealthy moles and 160 healthy moles for skin cancer lesions.

On the other hand, if the sensitivity of the experiments is captured as the most significant a part, it means that every patient with skin cancer is detected, and this method (ABC) is the best system in the comparison. So, it can be determined that the introduced method is a good and healthy decision to avoid false negatives when detection melanoma skin cancer. Table 2 shows the performance factors of the proposed method (\%) are used. To experimentally compare the implementation of the advanced segmentation algorithm with existing state of the art segmentation algorithms applying recognized statistical assessment parameters. The proposed $\mathrm{ABC}$ algorithm was confirmed on a two hundred challenging various benign and melanoma medical images obtained from PH2 databases.

\section{Conclusion}

In this paper, artificial bee colony established melanoma detection has proposed. Moreover, its computation time to detect a melanoma image is very fast. Since this detection is done in several steps where the specialist should do a careful analysis based solely on the information of the patients and their knowledge about the case. For comparison with the related works, it can be said that the proposed method obtained better results that the use of the databases of images were different. The methodology in this study was satisfactory, since, with the increase of the characteristics analyzed, the accuracy, sensitivity and specificity near may be reached at $100 \%$ success rate. Finally, the proposed method is more suitable for detecting melanoma in the skin. In near future this work will develop to detect different deep problems of skin not only cancer.

\section{References}

1. Cheng L, Wu XH, Wang YR (2018) Artificial Flora (AF) optimization algorithm. Appl Sci 8(3): 329.

2. Stoecker WV, Chiang CS, Moss RH (1992) Texture in skin images: Comparison of three methods to determine smoothness. Computerized medical imaging and graphics 16(3): 179-190.

3. Li L, Sun L, Guo J, Han C, Zhou J, et al. (2017) A quick artificial bee colony algorithm for image thresholding. Information 8(1): 16.

4. Casari A, Chester J, Pellacani GR (2018) Actinic keratosis and noninvasive diagnostic techniques: An update. Biomedicines 6(1): 8 .

5. Barata C, Celebi ME, Marques JS (2015) Improving dermoscopy image classification using color constancy. IEEE journal of biomedical and health informatics 19(3): 1146-1152.

6. Amelard R, Glaister J, Wong A, Clausi DA (2015) High-Level Intuitive Features (HLIFs) for intuitive skin lesion description. IEEE Transactions on Biomedical Engineering 62(3): 820-831.

7. Goyal M, Yap MH (2017) Multi-class semantic segmentation of skin lesions via fully convolutional networks.

8. Yuan Y (2017) Automatic skin lesion segmentation with fully convolutional-deconvolutional networks.

9. Al-Masni MA, Al-Antari MA, Choi MT, Han SM, Kim TS (2018) Skin lesion segmentation in dermoscopy images via deep full resolution convolutional networks. Computer methods and programs in biomedicine 162: 221-231.

10. Dey N, Rajinikanth V, Ashour AS, Tavares JM (2018) Social group optimization supported segmentation and evaluation of skin melanoma images. Symmetry 10(2): 51.

11. Li Y, Shen L (2018) Skin lesion analysis towards melanoma detection using deep learning network. Sensors 18(2): E556.

12. Guo Y, Ashour AS, Smarandache F (2018) A novel skin lesion detection approach using neutrosophic clustering and adaptive region growing in dermoscopy images. Symmetry 10(4): 119.

13. Abbes W, Sellami D (2017) Automatic skin lesions classification using ontology-based semantic analysis of optical standard images. Procedia Computer Science 112: 2096-2105.

14. Menegola A, Fornaciali M, Pires R, Bittencourt FV, Avila S, et al. (2017) Knowledge transfer for melanoma screening with deep learning. IEEE Transactions on Biomedical Engineering pp. 297-300.

15. Xie F, Bovik AC (2013) Automatic segmentation of dermoscopy images using self-generating neural networks seeded by genetic algorithm. Pattern Recognition 46(3): 1012-1019. 
16. Silveira M, Nascimento JC, Marques JS, Marçal AR, Mendonça T, et al. (2009) Comparison of segmentation methods for melanoma diagnosis in dermoscopy images. IEEE Journal of Selected Topics in Signal Processing 3(1): 35-45.

17. Karaboga D (2005) An idea based on honey bee swarm for numerical optimization. Research Gate.

ISSN: 2574-1241

DOI: 10.26717/BJSTR.2018.09.001789

Mohanad Hasan Ali Aljanabi. Biomed J Sci \& Tech Res

(c) (i) This work is licensed under Creative

Submission Link: https://biomedres.us/submit-manuscript.php
18. Mendonça T, Ferreira PM, Marques JS, Marcal AR, Rozeira J (2013) $\mathrm{PH}^{2}$-a dermoscopic image database for research and benchmarking. Conf Proc IEEE Eng Med Biol Soc 2013: 5437-5440.

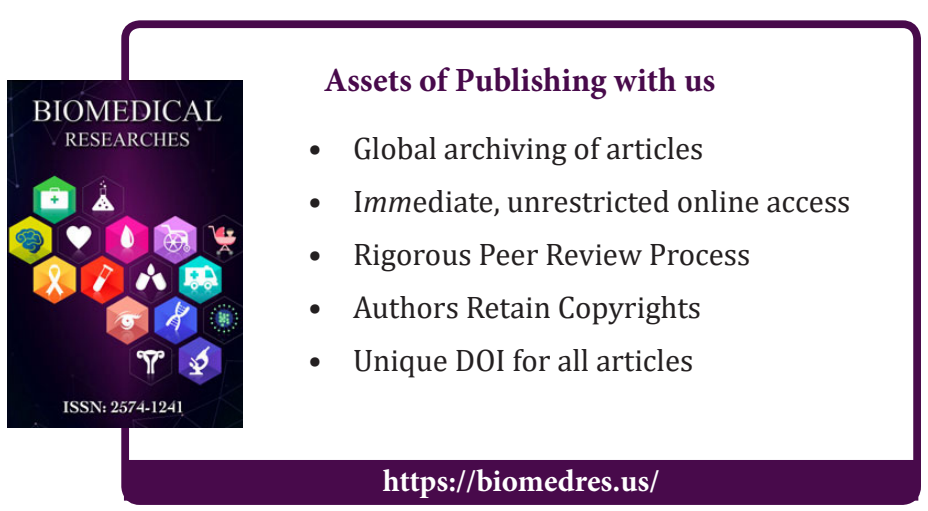

